\title{
REINFORCEMENT CONCORDANCE INDUCES AND MAINTAINS STIMULUS ASSOCIATIONS IN PIGEONS
}

\author{
JUAN D. DELIUS, MANUELA AMELING, STEPHEN E. G. LEA* \\ and J.E. R. STADDON ${ }^{+}$ \\ Universität Konstanz, Germany
}

\begin{abstract}
In a first experiment. pigeons were trained to discriminate two pairs of simultaneously presented stimuli. A+ C- and B+ D-. Both pairs were successively and repeatedly presented in every session. After the birds learned the two discriminations, both tasks were synchronously reversed (i.e., A- C+ and B-D+) several times. When reversal performance had stabilized, test reversal sessions were run in which one discrimination (the "leader" task, e.g., A+ C-) was presented for several trials before the second one (the "trailer" task, e.g., B+ C-) was introduced. The animals acquired the trailing task somewhat faster than the leading task, suggesting that associations $A \leftrightarrow B$ and $C \leftrightarrow D$ that had built up between the stimuli forming the two discrimination pairs were supporting a reversal transfer. A second experiment showed that further reversal experience with a discrimination where the constituent stimuli were presented compounded $(\mathrm{AB}+\mathrm{CD}-$ or $\mathrm{AB}-\mathrm{CD}+)$ as well as singly, enhanced the transfer between leading and trailing tasks in subsequent test sessions. A third experiment showed that the same pigeons learned half reversals involving only one discrimination (for example by switching from $A+B-, C+D-$ to $A-B+, C+D-)$ more slowly than full reversals involving both discriminations. These results support the hypothesis that pigeons can associate stimuli that have concordant reinforcement histories. When a reinforcement allocation change causes a change in responding to one stimulus of such an association, pigeons tend to generalize that response change to the other stimulus.
\end{abstract}

Part of the research, done while the authors were at the Psychology Institute, University of Bochum, was preliminarily presented in Ameling (1987). We are grateful to A. Lohmann for essential assistance with the experiments, to Dr. J. Emmerton for a computer program and improving an early draft, and to A. Niemuth for help with the manuscript. While being engaged in this research S. E. G. Lea and J. E. R. Staddon held Alexander von Humboldt awards. The work was supported by a Deutsche Forschungsgemeinschaft grant to J. D. Delius. Present addresses: ${ }^{\star}$ Department of Psychology, University of Exeter, Exeter EX4 4QG, England, +Department of Psychology: Experimental, Duke University, Durham NC 27706, USA. All correspondence to J. D. Delius, Allgemeine Psychologie, Universität Konstanz, D 78434 Konstanz, Germany. 
Although the old dispute about whether or not conditioning leads to a strengthening of stimulus-stimulus or of stimulus-response connections has generally been resolved by conceding that it can do both, specific questions remain. What precise conditions favor the buildup of stimulusstimulus associations, for example? The more cognitive branches of learning research would probably profit much from precise answers to this question. Indeed. the suggestion that interstimulus associations may be the essential constituents of so-called perceptual concepts (Lea, 1984) gave the initial impetus to the present study. Here, however, we begin with some simpler facts.

In a series of experiments Rescorla conditioned rats with dissimilar stimulus elements compounded into a single stimulus predicting reinforcement. He was able to show that the animals had associated the elements in a manner that was at least partly independent of the specific acquisition context (Rescorla, 1981; see also Pearce \& Wilson, 1990). Both the circumstances that the elements were presented in close contiguity and that each element signaled the same reinforcement could, however, have been instrumental in the formation of the interelement association. Other experiments (Bateson \& Chantrey, 1972; also Chantrey, 1972) using chickens and monkeys, however, have shown that simple exposure to stimulus contiguity alone, without reinforcement, can be sufficient to induce associations of a similar nature.

The question that remains is whether or not stimuli that signal the same reinforcement outcome, but are not themselves contiguous, can become associated in a similar manner. Lea (1984) suggested procedures capable of revealing stimulus associations of this kind. The simplest version first arranges that two stimuli $A$ and $B$ are consistent predictors of reward and that two stimuli $C$ and $D$ are predictors of nonreward. When the subjects' behavior matches these conditions, the reinforcement contingencies within a pair of stimuli of each kind, say $A$ and $C$ are reversed until the subjects have adapted again. The requisite association(s) between $A$ and $B$ and/or $C$ and $D$ would be demonstrated if on subsequent presentation of stimulus pair $B D$, subjects spontaneously responded to them in reversed manner too. The procedure is a modification of a partial-reversal design with which Sanders (1971; see also Kendler \& Kendler, 1968) showed that older children, but apparently not rats and younger children, could partially transfer reversed responding from one discrimination to another.

Partial-reversal designs of this general kind have been used in attempts to clarify the role of interstimulus associations in discriminations of the category or concept type. Vaughan (1988) had pigeons discriminate two sets of tree pictures. After repeated reversals they yielded reliable evidence of reversal transfer. Lea, Ryan, and Kirby (1990) had pigeons discriminate groups of letters and upon a single reversal obtained some evidence of reversal transfer. In contrast, Fersen and Lea (1990) trained pigeons to distinguish two sets of townscape pictures involving several binary features. When they were reversed with 
respect to one of these features the animals showed no evidence of reversal transfer to the other features. More recently Zentall, Steirn, Sherbourne, and Urcuioli (1991) again obtained some evidence of reversal transfer in pigeon experiments involving both straight and conditional discrimination paradigms. The reasons for these different outcomes are not clear because the procedures used differed in several respects. The precise conditions for the occurrence of reversal transfer in this species obviously still need further exploration. The present study reports experiments aimed at confirming whether pigeons could acquire stimulus associations in very simple contexts.

\section{Experiment 1}

Pigeons learned to discriminate concurrently two pairs of simple stimuli where responses to one stimulus of each pair were rewarded and the responses to the other were not rewarded $(A+C-, B+D-)$. When the subjects achieved discrimination, the reinforcement allocations to the stimuli were synchronously reversed $(A-C+, B-D+)$. As soon as discrimination was attained again, reinforcement allocations were reversed anew $(A+C-, B+D-)$, and so repeatedly. When reversal performance had stabilized, test sessions were run. In these sessions reversals began with the repeated presentation of only one pair of stimuli, the other pair being presented only after a lag of several trials (for example: $A+C-, A+C-, \ldots, A+C-, A+C-, B^{*} D^{*}, B^{*} D^{*}, B+D-, \ldots$ where the symbol * marks special, nonreinforced trials). The expectation according to the association hypotheses was that reversal performance acquired with the leading pair $(A C)$ discrimination would facilitate the subsequent reversal learning of the trailing pair $(B D)$.

Methods

Subjects. Eight adult homing pigeons (Columba livia) of local origin took part in the experiment. The birds were maintained at approximately $85 \%$ of their normal weights throughout the experiment and were kept in individual cages in a brightly lit (lights $12 \mathrm{~h}$ on, $12 \mathrm{~h}$ off) and wellventilated animal room.

Apparatus. The conditioning chamber measured $32 \times 32 \times 30 \mathrm{~cm}$. One of its walls bore two translucent keys $(2.5-\mathrm{cm}$ diam., $7 \mathrm{~cm}$ between centers, $20 \mathrm{~cm}$ above the floor). White, or red and green, or yellow and blue lights were back-projected onto the keys with an automatic slide projector. The projector took special slides with perforations that corresponded to the two keys on the chamber. As required the perforations were covered with clear or colored cellophane. The opening of a solenoid-operated feeder was centered below the keys $7 \mathrm{~cm}$ above the floor. All events within a session were controlled by an on-line computer system (Xia, Wynne, Münchow-Pohl, \& Delius, 1991) that also recorded all relevant data on a trial-by-trial basis.

Pretraining. The subjects were conditioned to peck the keys for grain 
using white light stimuli with an autoshaping program (5 s stimuli, $3 \mathrm{~s}$ food, 40 s interval) during six 30 min sessions.

Discrimination 1. The daily discrimination training sessions consisted of 32 trials. A trial began with red and green lights being projected on the keys. When the animal pecked the green key, the stimuli extinguished, food was offered for $3 \mathrm{~s}$. The next trial began when the projector had advanced to the next slide (equivalent to a $3-s$ intertrial interval). The left-right positions of the red and green stimuli were determined by a quasi-random sequence (Gellermann, 1933). If in a given trial the subjects responded to the negative stimulus, the stimuli and the houselights extinguished for $6 \mathrm{~s}$ (time-out). The same slide was then projected again; this correction procedure only ended when the subject made a correct, rewarded choice. The choices made while this procedure operated were not taken into account for performance scoring purposes.

Reversals. Subjects reached a criterion of $90 \%$ correct (green) choices on Session 4. Session 5 incorporated a reversal; responses to the red key were rewarded, those to the green key were not rewarded. After the birds had reached the criterion on this reversal task they were reversed again on Session 9 . This procedure was continued from then onwards except that for reasons of expediency, the criterion for reversals was reduced to $75 \%$ correct. A modified fixed ratio schedule was introduced on Session 14, so that within a trial only $N$ consecutive responses on the correct color led to reinforcement, whereas $N$ not necessarily consecutive responses on the incorrect color led to nonreward. A trial was counted as correct when it terminated with reward, and as incorrect when it ended with time-out. $N$ was gradually increased up to six and this value was retained from Session 18 onwards. By this session the birds had undergone eight reversals.

Discrimination 2. Starting with Session 19, half of the $32 \mathrm{red} / \mathrm{green}$ slides were replaced by blue/yellow slides. The sequence of the two kinds of trials within a session was quasi-random (Gellermann, 1933). Reversals continued as before, but with reinforcement allocations always yoked across both discriminative tasks. Whenever responding to red was rewarded, responding to blue was also rewarded. Conversely, whenever green was rewarded, yellow was also rewarded. This was expected to foster associations between the corresponding pairs of colors. From Session 34 onwards reversal sessions were scheduled independently of performance quasi-randomly on either the first, second, or third session after the last reversal. Beginning with Session 76 there were four (two per discrimination task) extinction trials in each session. Schedule completion (six not necessarily consecutive responses to one or the other of the two stimuli) during these trials led neither to reward nor to time-out but directly to the next trial. These extinction trials (their purpose will become apparent soon) were randomly inserted among normal trials avoiding the first three and last three of any session. Until the final training Session 86 . the birds had undergone a total of 34 both-discrimination reversals. 
Tests. The 24 sessions that followed included 12 test reversal sessions. Within these latter the sequence of $16 \mathrm{red} / \mathrm{green}$ trials and 16 blue/yellow trials was not quasi-random as the previous reversal sessions. Rather, for each such session, one of the stimulus pairs (for instance $\mathrm{red} / \mathrm{green}$ ) was designated to be the leading reversal pair and the other (blue/yellow in the example) to be the trailing reversal pair according to an overall balanced randomized design. The first 8 to 12 trials of these test sessions were leading pair trials only (e.g., all red/green). A random sequence of trailing pair trials (e.g., blue/yellow) and remaining leading trials (e.g., red/green) followed. Two randomly selected leading-type trials and, importantly, the first two trailing trials were nonreinforced.

\section{Results and Discussion}

The development of the discriminative performance during the various phases of training and during testing was assessed by calculating average percentage of trials correct per session across the subjects. These scores did not include correction trials, but included nonreinforced trials. The extinction trials were counted as correct when the subjects chose the stimulus that normally would have led to reward. As was expected, performance improved with the animals' increasing experience. This applied particularly to the average scores achieved during reversal sessions. At the end of the training phase, they invariably oscillated above the $75 \%$ correct choices level.

The subjects' performance during the test reversals was evaluated by calculating mean percentage of choice scores across the relevant sessions on a trial-by-trial basis, separately for the leading and trailing components. The crucial data are the performance scores on the initial trials of each component task, taking into account the design details explained earlier in the Methods section. That is, stimulus choices during only the first leading trial of each test session were uninfluenced by preceding reinforcement feedback while, at the same time, stimulus choices during the first three trailing trials of the same sessions were free from such feedback (i.e., the two initial extinction trials and the first reinforced trial).

Table 1 presents the mean performance scores of each bird computed

Table 1

Mean Percentage of Correct Choices During First Leading and First Trailing Trials in 12 Test Reversals (Exp. 1)

\begin{tabular}{ccc}
\hline Pigeon & Leading & Trailing \\
\hline 39 & 33.3 & 33.3 \\
46 & 41.6 & 77.8 \\
54 & 41.6 & 50.0 \\
61 & 50.0 & 33.3 \\
64 & 41.6 & 55.5 \\
68 & 33.3 & 36.1 \\
86 & 25.0 & 36.1 \\
98 & 33.3 & 63.9 \\
Averages & 37.4 & 48.3 \\
\hline
\end{tabular}


on that basis. As predicted by the transfer hypothesis, the somewhat better average first trailing trials versus first leading trials performance is just significant $(p=0.05$, permutation test for paired alternatives, Siegel \& Castellan, 1988). Because the pigeons required an average 6.0 reinforced trials on the leading component to reach a mean two-trial, near-asymptote level of $87.5 \%$ correct, it is obvious that when the trailing component was introduced about 10 trials into the test sessions, the leading discrimination was already being solved with near maximum accuracy. Again in accordance with the transfer hypothesis, the pigeons needed significantly less trials to achieve the same criterion on the trailing component (an average 4.3 reinforced trials; $p<0.05$ ).

Although there is a significant double advantage of the trailing over the leading task, the effect is not large. Furthermore we can not ascribe it with certainty to the transfer process implied earlier. A simpler account could be that the experience with the reversal of the leading task causes the subjects to revert to a nondiscriminative strategy when confronted with the trailing task. An analogous signaled extinction responding is assumed to operate when reversal learning is progressively facilitated by the institution of regularly, rather than randomly, scheduled reversals (Staddon \& Frank, 1974). The hypothetical conditional extinction explanation would accord with the fact that the initial trailing discrimination scores were at chance level (average $48.3 \%$, Table 1). In turn, this neutral beginning can be imagined to have facilitated the subsequent acquisition of the trailing task, particularly if an intrasession learning warm-up effect would have added to it. Note that this account implies that the trailing task advantage effect would only have gradually built up as the subjects gained experience with test reversals. However, a comparison of the results that arose during the first and the second half of the test sessions does not reveal any significant differences. Nevertheless, we shall return to this issue later.

\section{Experiment 2}

The preceding experiment yielded evidence of a small but significant reversal facilitation between the discrimination tasks. The experiment we now describe included further discrimination reversal training as before, but also attempted to induce stronger associations between the relevant stimuli by additionally presenting them in spatial contiguity. The four discriminative stimuli thus became, in effect, elements of a single pair of compound stimuli ( $A B$ and $C D$ ). These compound pairs were presented in trials interspersed among simple stimuli pairs (for example, A+C-, AB+CD-, B+C-,...). The subjects could thus be expected to form within-compound associations (Rescorla, 1981). During reversal transfer testing using a leading-trailing procedure much as in the previous experiment, these compound stimuli were, however, all relegated to the noncritical final third of each of the test sessions.

\section{Methods}

Subjects and apparatus. They were the same as those used in Experiment 1. 
Training. The procedure was identical to that previously followed except that within a session 12 slides (replacing 6 of the 16 red/green and 6 of the 16 blue/yellow slides used in the previous experiment) involved twocolor compound stimuli: red-blue/green-yellow. During the corresponding trials, one key was thus illuminated half with red and half with blue light, whereas the other was illuminated half with green and half with yellow. Which color filled the left half and which the right half of a given key was quasi-random (Gellermann, 1933). Which color combination appeared on which key was also quasi-randomly determined. Schedule completion on the keys bearing these compound stimuli led to reward or to time-out in the same way and always coherently with the outcome programmed for the two simple stimulus pairs. Within each session the three kinds of trial were randomly sequenced. There were 20 training sessions including 10 quasirandomly scheduled reversals.

Test. The 34 sessions that followed included 16 test reversals. These latter were closely similar to those run in Experiment 1. The sessions began with six or seven leading-type (simple stimuli) trials. A random sequence of several trailing and leading (all simple stimuli) trials followed. The 12 compound trials stimuli were randomly inserted among the remaining simple stimuli during the uncritical last third of the sessions. Two randomly selected leading trials and, importantly, the two first trailing trials were not reinforced.

\section{Results and Discussion}

Results were assessed as before. The average performance during all the sessions (including reversal sessions) of this experiment oscillated above the $75 \%$ correct choices mark. The test reversal session data were analyzed in the same way as in Experiment 1 . The mean choice accuracy of the individual birds during the first leading and 3 first trailing trials of the test sessions is displayed in Table 2. The average initial performance advantage of the trailing discrimination component over the leading component is now definitely significant ( $p<0.05$, permutation test as before). The number of trials the pigeons needed to reach near-asymptote accuracy (two-trial mean of $87.5 \%$ correct choices) with trailing stimuli is very significantly less than with leading stimuli (average 6.1 versus 1.6 reinforced trials, $p<0.001$ )

By yielding a more sizeable reversal facilitation effect Experiment 2 provides stronger support for the stimulus association hypothesis than Experiment 1. Though the straightforward additional reversal experience preceding the tests could have contributed, it seems reasonable to assume that some of the effect improvement was a consequence of the use of compound stimuli during training. However, the stronger effect obtained in this experiment still does not quite exclude the conditional extinction account considered earlier, as the initial trailing task performance was still at chance level (average 49.1\%, Table 2). Through avoidance of the leading-trailing procedure the experiment that follows was intended to resolve this issue. 
Table 2

Mean Percentage of Choices Correct During First Leading and First Trailing Trials in 16 Test Reversal Sessions (Exp. 2)

\begin{tabular}{ccc}
\hline Pigeon & Leading & Trailing \\
\hline 39 & 31.1 & 35.4 \\
46 & 37.5 & 66.6 \\
54 & 31.1 & 52.0 \\
61 & 37.5 & 56.2 \\
64 & 37.5 & 43.8 \\
68 & 56.3 & 31.1 \\
86 & 31.1 & 45.8 \\
98 & 50.0 & 62.5 \\
Averages & 39.0 & 49.1 \\
\hline
\end{tabular}

\section{Experiment 3}

Although some evidence argued against a conditional extinction effect explanation, the design of the preceding experiments could not totally exclude it. The present experiment was expressly designed to control for it. The experiment compared the pigeons' performance on full-reversal sessions (both stimulus pairs reversed, from $A+C-, B+D-$ to $A-C+, B-D+$ for example) with that on half-reversal sessions (only one pair reversed, from A-C+, B- D+ to A+C-, B- D+ for example). The procedure was now in fact very similar to that used by Sanders (1971) and more recently by Nakagawa (1986) and Zentall et al. (1991). The expectation was that the full reversals, by being consistent with the stimulus linkages $A \leftrightarrow B$ and $C$ $\leftrightarrow \mathrm{D}$ that had been operative throughout the previous experiments, would be acquired faster than the half reversais, which involved a disruption of the previous corresponding associations. This contrasts strongly with the more naive expectancy that the learning of two discrimination reversals should be lengthier than the learning of just one.

No compound stimuli were used in this experiment. If the interstimulus associations posited depend strictly on stimuli occurring together as elements in compounds, one should now expect a full- over half-reversal advantage to gradually wane. But if the experience with compound stimuli during Experiment 2 had merely served to facilitate a process of association by reinforcement outcomes, then the full-reversal task advantage should be sustained.

\section{Methods}

Subjects and apparatus. They were the same as those used in Experiments 1 and 2.

Training. Six retraining sessions including four reversals were run. In view of the fact that the subjects' reversal performance was now excellent, the sessions were reduced to 20 trials, but all of these were reinforced. They involved the presentation of $10 \mathrm{red} / \mathrm{green}$ and 10 blue/yellow slides in quasi-random sequence (Gellermann, 1933). 
Tests. Included among 44 further sessions there were 8 test reversal sessions. The subjects were divided into two matched groups of about equal previous performance. As specified by a counterbalanced design, on any one of the test sessions one of the groups was exposed to a full reversal of both color discriminations and the other group to a half reversal, in which only one color discrimination was reversed but not the other discrimination. Regardless of this the red/green and blue/yellow trials were quasi-randomly sequenced throughout; the design no longer involved a leading-trailing differentiation nor any extinction trials. Each bird was accordingly tested in four full-reversal and four half-reversal sessions. In the session following these tests the half-reversal group reverted to the fullreversal condition, the remaining discrimination now also being reversed. They were thus then being treated exactly the same way as the fullreversal group was. Subsequently, and before the next test session, all birds underwent two quasi-randomly scheduled normal, full reversals. These were intended to redress any associational confusion that might have been caused by the transitory test half reversals.

Results and Discussion

As might be expected the new test reversal procedure led to a transitory drop in the birds' average session performance but this soon normalized and fluctuated at well above the $75 \%$ choices correct mark.

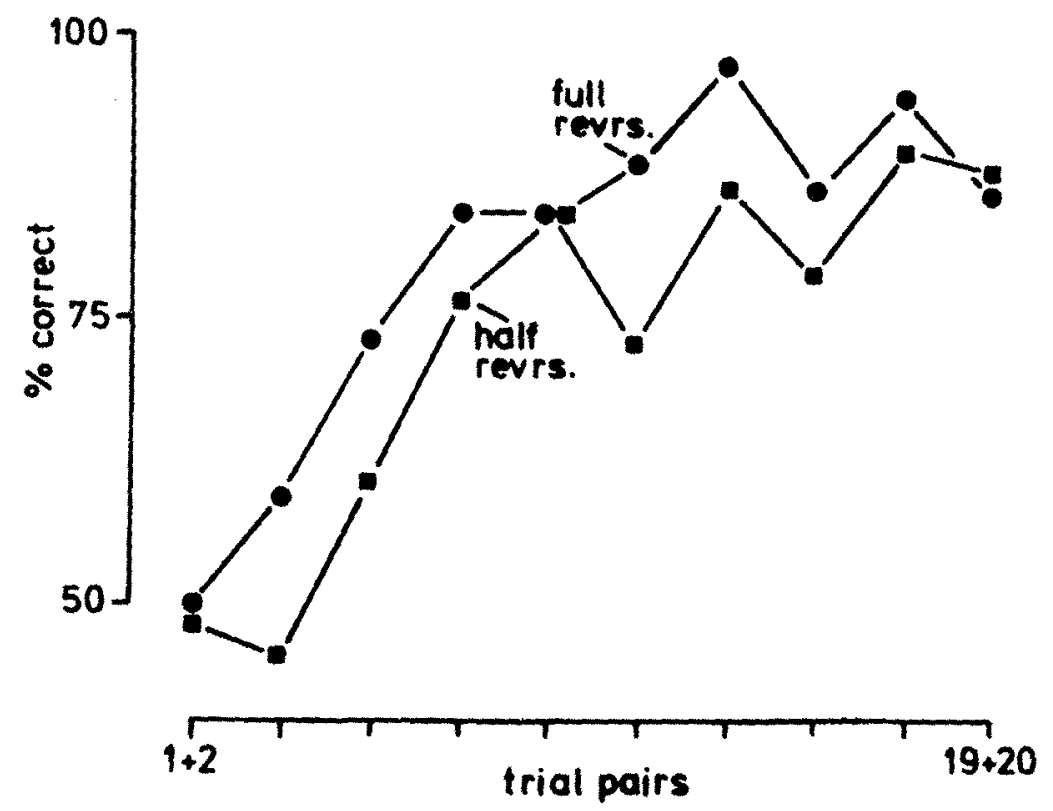

Figure 1. Average discrimination performance of pigeons $(n=8)$ during four fullreversal (circles) and four half-reversal (squares) sessions plotted as a function of the progression of trial pairs (Experiment 3). 
The mean discrimination accuracy of the eight birds was calculated on a trial by trial basis separately across the four full- and the four half-reversal test sessions. Correct choices were defined, as before, by whether they led to reward or not. For this initial evaluation no distinction was made between the component tasks, neither during full nor half reversals. Figure 1 presents the average learning progress during full- and half-reversal sessions as functions of blocks of two trials. It reveals a persisting poorer performance during the half-reversal sessions as compared with that during the full-reversal sessions, despite the fact that the pigeons had to relearn one discrimination in the former case and two in the latter case. Table 3 presents separately the mean percentage of trials correct that each bird accumulated during the first 10 trials (i.e., during the acquisition phase) in each kind of condition during the corresponding sessions. Performance in the full-reversal condition is significantly better than in the half-reversal condition ( $p=0.01$, permutation test). A cumulation of trials correct over all 20 trials yields a similar but less significant difference $(p<0.05)$ because of a ceiling effect that affects both conditions during the latter part of the sessions. The birds also needed fewer trials to reach near-asymptote

Table 3

Average and Mean Percentages of Choices Correct in Experiment 3

\begin{tabular}{|c|c|c|c|c|c|}
\hline \multicolumn{4}{|c|}{ All Data } & \multicolumn{2}{|c|}{ Only More-Train. Data } \\
\hline Pigeon & Half Rev. & Fulf Rev. & Training & Half Rev. & Full Rev. \\
\hline 39 & 62.5 & 67.5 & 82.5 & 60.0 & 75.0 \\
\hline 46 & 52.5 & 70.0 & 80.0 & 45.5 & 85.0 \\
\hline 54 & 65.0 & 65.0 & 85.0 & 65.0 & 85.0 \\
\hline 61 & 62.5 & 72.5 & 90.0 & 65.0 & 85.0 \\
\hline 64 & 70.0 & 67.5 & 97.5 & 65.0 & 60.0 \\
\hline 68 & 57.5 & 77.5 & 87.5 & 65.0 & 80.0 \\
\hline 86 & 70.0 & 72.5 & 87.5 & 65.0 & 90.0 \\
\hline 98 & 67.5 & 67.5 & 82.5 & 75.0 & 80.0 \\
\hline Averages & 63.4 & 70.0 & 86.6 & 63.1 & 80.0 \\
\hline
\end{tabular}

Note. Left (all data): Average percentages of choices correct during first 10 trials of all 4 half-reversal test sessions, all 4 full-reversal test sessions, and 4 randomiy selected training sessions. Right (only more-training data): Mean percentages of choices correct of two half-reversal and two full-reversal test sessions that were preceded by more than average training sessions (see text, Experiment 3 ).

performance $(87.5 \%$ correct) in the full-reversal condition $(4.7$ against 6.0 trials, $p=0.05$ ). The pigeons obviously readjusted faster to the full-reversal condition than to the half-reversal condition. This is in accordance with the reversal transfer expected under the stimulus association hypothesis and contrary to the naive expectation that one discrimination reversal should be easier to master than two such reversals. An explanation of the difference in terms of the conditional extinction mechanism considered earlier does not apply here, as both types of reversals begin identically at chance level (Fig. 1, Trials 1 and 2). In the same context, an analysis that distinguished between the nonreversed and reversed components of the half-reversal condition failed to reveal any significant differences. 
For comparison, Table 3 (third column) also presents the average discrimination performance of the same pigeons during the 10 first trials of 4 randomly selected, normal training sessions not involving any reversal. The performance here is clearly better than that under the fullreversal condition $(p<0.005)$. The pigeons obviously carried over a definite and persisting preference for the stimuli that had been positive during the preceding sessions.

A further analysis showed that there was no decline in the fullversus half-reversal advantage during the experiment but that, on the contrary, the effect increased somewhat, although not significantly, as it progressed. This indicates that the association between the relevant stimuli (red/blue and green/yellow) was maintained by reinforcement concordance alone even when the compound stimuli of Experiment 2 were no longer in use.

A partition of the test sessions into those that were preceded by one or two, rather than by no, training sessions since the last routine reversal reveals that additional prereversal training importantly influences the performance difference between full and half reversals. For the moretraining data (Table 3, right), the advantage of full over half reversals is significant $(p<0.005)$. The less-training data (not shown), if anything, reveal a slight contrary but not significant trend (average half reversals $63.7 \%$, average full reversals $60.0 \%$ correct). The difference caused by more versus less training is in itself significant $(p=0.01$, permutation test). This finding agrees with Nakagawa's $(1986 ; 1992)$ finding that additional prereversal training promotes the occurrence of the fullreversal versus half-reversal advantage in rats.

\section{General Discussion}

Taken together, the results of all three experiments provided evidence of a partial transfer of reversal performance from one twostimulus discrimination to the other. Stimuli that during training were operationally linked by concordant reinforcement outcomes appeared to be associated by the animals in a way that transcended the specific acquisition situation. When the pigeons learned that the outcome of one such stimulus had changed they partly transferred that knowledge to the other stimulus. The transfer effects obtained, though not excessively strong, were all significant. Furthermore it is worth pointing out that one subject (Pigeon 46) consistently exhibited marked effects across all three experiments (Tables 1, 2, 3).

A good reversal learning performance is an obvious operational precondition for a sizeable transfer of reversal performance. Repeated reversals are known to improve the acquisition of discrimination reversal in pigeons as well as other animals (Beale, 1970; Staddon \& Frank, 1974). Indeed, our pigeons also exhibited some improvement of reversal performance with increasing reversal experience. The increasing magnitude of the transfer effect noted across our experiments is likely to 
be at least partly caused by that improvement in reversing skill. Experiments 2 and 3 of Zentall et al. (1991) using pigeons and a design similar to that of our Experiment 3, except for the employment of a successive discrimination method, yielded results that also conform with this assumption. Vaughan's (1988) marked success in demonstrating reversal transfer in pigeons using again a successive paradigm but employing many complex stimuli is very probably partially because of the repeated reversal design that he used (Fersen \& Lea, 1990). Sidman, Wynne, Macguire, and Barnes (1989), in any case, found that they needed to implement repeated reversals before they could obtain evidence of stimulus association in mentally defective humans. It is conceivable, however, that the employment of a successive, rather than simultaneous discrimination procedure would also tend to yield stronger evidence of reversal transfer, perhaps because it is less likely to invite a solution strategy based on stimulus-pair configurations. The within-pair associations that this implies can be easily imagined to interfere with the between-pair associations one is seeking to demonstrate.

It is known that an overtraining of the preceding discrimination tends to enhance the acquisition of the succeeding reversal. This so-called overtraining reversal effect has also been demonstrated in pigeons (Beale, 1970; Williams, 1967). Nakagawa (1986, see also Nakagawa, 1992) attributes her successful demonstration of reversal transfer in rats with a single reversal to the enforcement of overtraining. Indeed, Sanders (1971) using an otherwise similar design but no overtraining could not find transfer in rats, nor indeed in younger children. A post-hoc analysis of our Experiment 3 results indicates that even a moderately extended training previous to reversals strongly enhances the reversal transfer effect in pigeons. However, this insight came too late for what, in retrospect, could have meant an improvement in experimental design.

One can not be certain that the spatial contiguity between stimuli introduced during training for Experiment 2 was actually responsible for the somewhat stronger stimulus association effects obtained afterwards. These effects could also have been caused by the increasing discrimination and reversal experience of the birds. However, as mentioned in the introduction, there are findings that support the view that plain contiguity can bring about interstimulus associations. Although Stewart, Capretta, Cooper, and Littlefield (1977) have questioned the robustness of the relevant evidence, there is no doubt that prior contiguous exposure to stimuli can yield substantial associative effects (Channel \& Hall, 1981; Pearce \& Wilson, 1990). All this suggests that the introduction of stimulus compounding in our experiments may after all have enhanced the occurrence of reversal transfer effects, much as would have been deduced from Rescorla's (1981) results. Although contiguity might have yielded a strengthening effect, the results of Experiment 3 showed that concordant reinforcement clearly sufficed for the maintenance of the stimulus associations underlying the transfer.

Even though strict comparison is not possible because of many 
procedural differences, Vaughan (1988) has undoubtedly obtained the strongest reversal transfer effects so far. We suggest the important variable responsible for this success may be the kind of stimuli that he used. Vaughan's complex pictures are likely to have provided the pigeons with more cues for the formation of interstimulus associations than our plain patches of color. Being slides of natural scenes they are likely to have contained several stimulus features in suitable contiguity within the stimulus sets, and even in adequate contingency across the stimulus sets. These uncontrolled factors, although possibly favoring interstimulus association, unfortunately also impede a precise analysis (compare Fersen \& Lea, 1990).

Regardless of interpretative details, there is now little doubt that pigeons possess the basic mechanisms that enable them to associate stimuli on the basis of reinforcement concordance. This in principle should also enable them to acquire and to maintain perceptual concepts based on interstimulus associative clusters in the sense specified by Lea (1984) and sketched earlier. However, it is important to note that the stimulus association mechanisms postulated are not necessarily in conflict with categorization processes based on simple similarity between stimuli (Bhatt \& Wasserman, 1989; Fersen \& Lea, 1990; Vaughan \& Herrnstein, 1987). On the contrary, it seems likely that in nature both principles may often act in consonance.

As pointed out, stimulus-stimulus associations of the kind addressed here seem to be essential for the formation of so-called perceptual concepts. Such concepts can be conceived as intervening constructs that bundle into groups many stimuli that control responding in a very similar manner. Perceptual concepts in turn are basic elements of cognitivistic accounts of behavior. There is good reason to believe that associative structures of this type tend to improve the informationprocessing efficiency of nervous systems. In nature concepts may often correspond to ecological entities such as niche, partners, predators or food, all thought to be crucial agents in the behavioral evolution of organisms. Associations between stimuli thus merit further and intensive behavioristic analysis.

\section{References}

AMELING, M. (1987). Gruppenzugehörigkeit von visuellen Reizen als Diskriminationsgrundlage bei Tauben (Group-belongingness of stimuli as a discriminative basis in pigeons). Diplom thesis, Bochum University.

BATESON, P. P. G., \& CHANTREY, D. F. (1972). Retardation of discrimination learning in monkeys and chicks previously exposed to both stimuli. Nature, $237,173-174$.

BEALE, I. L. (1970). The effects of amount of training per reversal on successive reversals of a color discrimination. The Journal of the Experimental Analysis of Behavior, 14, 345-352. 
BHATT, R. S., \& WASSERMAN E. A. (1989). Secondary generalization and categorization in pigeons. The Journal of the Experimental Analysis of Behavior, 52, 213-224.

CHANNEL, S., \& HALL, G. (1981). Facilitation and retardation of discrimination learning after exposure to the stimuli. Journal of Experimental Psychology: Animal Behavior Processes, 7, 437-446.

CHANTREY, D. F. (1972). Enhancement and retardation of discrimination learning in chicks after exposure to discriminanda. Journal of Comparative and Physiological Psychology, 81, 256-261.

FERSEN, L., von, \& LEA, S. E. G. (1990). Category discrimination with polymorphous features. The Journal of the Experimental Analysis of Behavior, 54, 69-84.

GELLERMANN, L. W. (1933). Chance orders of alternating stimuli in visua discrimination experiments. Journal of Genetic Psychology, 42, 206-208.

KENDLER, H. H., \& KENDLER, T. S. (1968). Mediation and conceptual behavior. In K. W. Spence \& J. T. Spence (Eds.), The psychology of learning and motivation (Vol. 2, pp. 197-244). New York: Academic Press

LEA. S. E. G. (1984). In what sense do pigeons learn concepts? in H. L. Roitblat, T. G. Bever, \& H. S. Terrace (Eds.), Animal cognition (pp. 263276). Hillsdale, NJ: Erlbaum.

LEA, S. E. G., RYAN. C. M. E., \& KIRBY, R. M. (1990). Instances to category generalization following pigeons' learning of an artificial concept discrimination. Internal Report, Animal Psychology Research Group, Department of Psychology, University of Exeter, 90/1, 1-38.

NAKAGAWA, E. (1986). Overtraining, extinction and shift learning in a concurrent discrimination in rats. Quarterly Journal of Experimental Psychology, 38B, 313-326.

NAKAGAWA, E. (1992). Effects of overtraining on reversal learning by rats in concurrent and single discriminations. Quarterly Journal of Experimental Psychology, 44B, 37-56.

PEARCE, J. M., \& WILSON, P. N. (1990), Configural associations in discrimination learning. Journal of Experimental Psychology: Animal Behavior Processes, 16, 250-261.

RESCORLA, R. A. (1981). Simultaneous associations. In P. Harzem \& M. D. Zeiler (Eds.), Advances in analysis of behavior, Vol. 2: Predictability, correlation and contiguity (pp. 47-80). Chichester: Wiley.

SANDERS, B. (1971). Factors affecting reversal and nonreversal shifts in rats and chiidren. Journal of Comparative an Physiological Psychology, 74 192-202.

SIDMAN, M., WYNNE C. K., MACGUIRE, R. W., \& BARNES, T. (1989) Functional classes and equivalence relations. The Journal of the Experimental Analysis of Behavior, 52, 261-274.

SIEGEL, S., \& CASTELLAN, N. J. (1988). Nonparametric statistics for the behavioral sciences. New York: McGraw Hill.

STADDON, J. E. R., \& FRANK, J. (1974). Mechanisms of discrimination reversal in pigeons. Animal Behavior, 22, 802-828.

STEWART, D. J., CAPRETTA, P. J., COOPER, A. J., \& LITTLEFIELD, V. M. (1977). Learning in domestic chicks after exposure to both discriminanda. Journal of Comparative and Physiological Psychology, 91, 1095-1109.

WILLIAMS, D. I. (1967). The overtraining reversal effect in the pigeon. Psychonomic Science, 7, 261-262. 
VAUGHAN, W. (1988). Formation of equivalence sets in pigeons. Journal of Experimental Psychology: Animal Behavior Process, 14, 36-42.

VAUGHAN, W., \& HERRNSTEIN, R. J. (1987). Choosing among natural stimuli. The Journal of the Experimental Analysis of Behavior, 47, 5-16.

XIA, L., WYNNE C. D., von MÜNCHOW-POHL, F., \& DELIUS, J. D. (1991). Psychobasic: A Basic dialect for the control of psychological experiments with the Commodore-64 and Dela interfacing. Behavioral Research Methods, Instruments \& Computers, 23, 72-76.

ZENTALL, T. R., STEIRN, J. N., SHERBOURNE, L. M., \& URCUIOLI, P. J. (1991). Common coding in pigeons assessed through partial versus total reversals of many-to-one conditional and simple discriminations. Journal of Experimental Psychology: Animal Behavior Processes, 17, 194-201. 